\title{
An Origin of Complicated Infrared Spectra of Perfluoroalkyl Compounds Involving a Normal Alkyl Group
}

\section{$\operatorname{AUTHOR}(\mathrm{S}):$}

Hasegawa, Takeshi; Shimoaka, Takafumi; Tanaka, Yuki; Shioya, Nobutaka; Morita, Kohei; Sonoyama, Masashi; Amii, Hideki; Takagi, Toshiyuki; Kanamori, Toshiyuki

\section{CITATION:}

Hasegawa, Takeshi ...[et al]. An Origin of Complicated Infrared Spectra of Perfluoroalkyl Compounds Involving a Normal Alkyl Group. Chemistry Letters 2015, 44(6): 834-836

\section{ISSUE DATE:}

2015-06

URL:

http://hdl.handle.net/2433/218806

\section{RIGHT:}

(c) The Chemical Society of Japan; この論文は出版社版でありません。 引用の際には出版社版をご確認ご利用ください。; This is not the published version. Please cite only the published version. 


\title{
An Origin of Complicated Infrared Spectra of Perfluoroalkyl Compounds Involving a Normal Alkyl Group
}

\author{
Takeshi Hasegawa* ${ }^{1}$, Takafumi Shimoaka ${ }^{1}$, Yuki Tanaka ${ }^{1}$, Nobutaka Shioya ${ }^{1}$, Kohei Morita $^{2}$, Masashi Sonoyama ${ }^{2}$, Hideki Amii $^{2}$, \\ Toshiyuki Takagi ${ }^{3}$, Toshiyuki Kanamori ${ }^{3}$ \\ ${ }^{1}$ Institute for Chemical Research, Kyoto University, Uji, Kyoto 611-0011, Japan \\ ${ }^{2}$ Division of Molecular Science, Faculty of Science and Technology, Gunma University, Kiryu, Gunma 376-8515, Japan \\ ${ }^{3}$ National Institute of Advanced Industrial Science and Technology (AIST), AIST Tsukuba Central 5, 1-1-1 Higashi, Tsukuba, Ibaraki 305- \\ 8565, Japan
}

(Received $<$ Month $><$ Date $>$, $<$ Year $>$; CL- $<$ No $>$; E-mail: htakeshi@scl.kyoto-u.ac.jp)

Perfluoroalkyl (Rf) compounds involving a normal alkyl group often yield highly complicated infrared (IR) spectra especially in the $\mathrm{C}-\mathrm{F}$ stretching vibration $(\mathrm{v}-\mathrm{F})$ region. To reveal the reason, the IR p-polarized multiple-angle incidence resolution spectrometry (pMAIRS) is employed to measure a monolayer of $\mathrm{CF}_{3}\left(\mathrm{CF}_{2}\right)_{9}\left(\mathrm{CH}_{2}\right)_{3} \mathrm{COOH}$ deposited on a silicon substrate. The compound is known to spontaneously aggregate to form a molecular assembly with a closest packing, in which the molecules are oriented perpendicularly to the substrate. The IR pMAIRS spectra apparently prove that the complexity of the $\mathrm{vC}-\mathrm{F}$ region is due to the normal alkyl part directly connected to the $\mathrm{Rf}$ group because the carbons in the $\mathrm{Rf}$ group are vibrated as a coupled oscillator, and the oscillation of the alkyl part propagates to the Rf part along the molecular axis.

A perfluoroalkyl (Rf) group is a fluorine-substituted alkyl group on all hydrogen atoms. When the whole or a portion of an alkyl group is replaced by an $\mathrm{Rf}$ group, the compound often exhibits an Rf-specific bulk character ${ }^{1}$ represented by the water/oil repellency, a low electric permittivity, and high melting point, which depends on the Rf length. ${ }^{2}$ To make a chemically functionalized material, Rf compounds have long been used in various chemical products, and understanding of the relationship between the primary chemical structure and the bulk property is necessary. ${ }^{3}$

Since an Rf group has a similar chemical structure to a normal alkyl group except the Rf group-specific molecular twist (Figure 1), the structure analysis of Rf compounds is performed by using an infrared (IR) spectroscopic technique, in which the trifluoromethyl $\left(-\mathrm{CF}_{3}\right)$ and difluoromethylene ($\mathrm{CF}_{2}-$ ) groups are expected to exhibit similar IR bands to those of the normal methyl and methylene groups, respectively. ${ }^{4}$

In a recent study, ${ }^{3}$ however, it is first pointed out that the $\mathrm{CF}_{2}$ stretching vibration bands cannot be used for discussing the molecular orientation of the Rf group, since an $\mathrm{Rf}$ group is twisted with a pitch depending on the phase diagram. ${ }^{5,6}$ When an Rf group has a tilted stance to the substrate surface, one of the $\mathrm{CF}_{2}$ groups could be parallel to the surface; whereas the rest $\mathrm{CF}_{2}$ groups should obliquely be aligned with different tilt angles because of the twisted molecular structure. ${ }^{3}$ Since a normal alkyl group has a planar zigzag structure in a crystalline, the situation of an Rf group is significantly different from that of the normal alkyl group. As shown straightforwardly by this example, the spectroscopic discussion of an Rf group and normal alkyl one should be discriminated from each other.

Another notable point to discuss IR spectra of $\mathrm{Rf}$ compounds is that the mass of fluorine is larger than that of

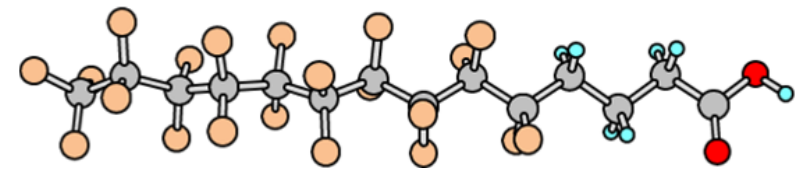

Figure 1 A schematic of the compound of $n=9$ with $r=3.0$. The $R f$ part is twisted about the molecular axis. The gray, orange, blue and red circles denote $\mathrm{C}, \mathrm{F}, \mathrm{H}$ and $\mathrm{O}$ atoms, respectively.

carbon, which is totally opposite to the $\mathrm{C}-\mathrm{H}$ bond: hydrogen is much lighter than carbon. As a result, on a molecular vibration, the fluorine atoms stay with nearly no motion; whereas the skeletal carbon chain vibrates as a coupled oscillator. In other words, a normal mode is not highly localized on a chemical group, ${ }^{7}$ but it is readily influenced by the connected groups in the direction of the molecular axis.

To systematically discuss IR spectra of Rf compounds in terms of the coupled oscillator, a series of di-block carboxylic acids consisted of the $\mathrm{Rf}$ and the normal alkyl parts are

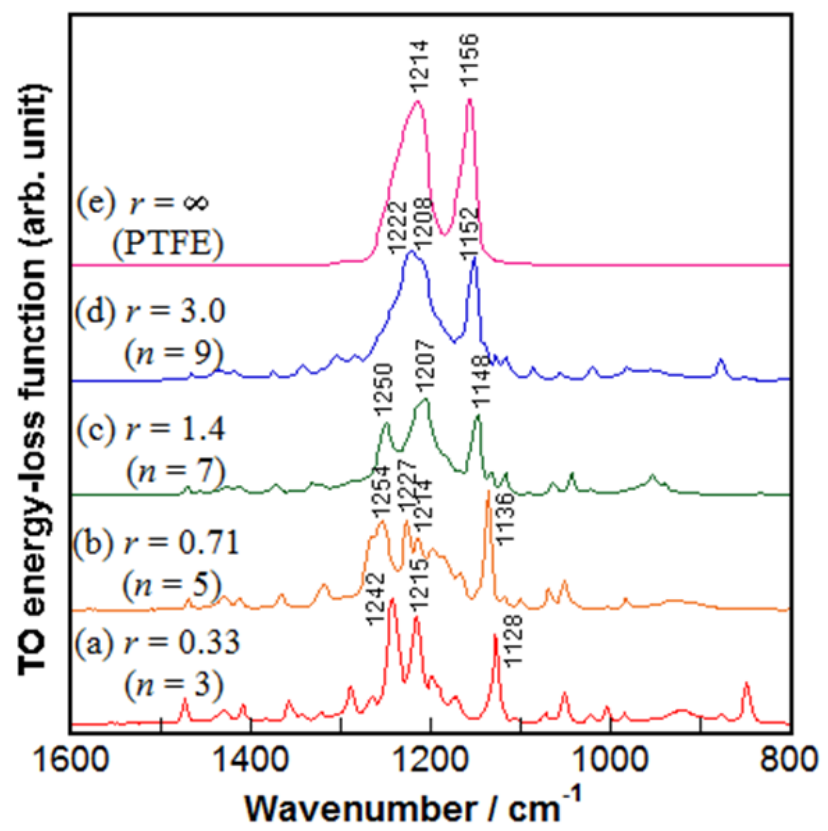

Figure 2 TO energy-loss function spectra of perfluoroalkyl-containing compounds with different length ratio, $r$, of the $\mathrm{Rf}$ group to the normal alkyl group. 
prepared, which is denoted as: $\mathrm{CF}_{3}\left(\mathrm{CF}_{2}\right)_{n}-\left(\mathrm{CH}_{2}\right)_{m}-\mathrm{COOH}$ (Figure 1). The total length is fixed at a constant $(n+m=12)$, so that the length ratio defined as $r \equiv n / m$ is introduced as a single parameter. The carboxylic group is introduced to make the melting point appropriate for the experiments in an ambient condition; otherwise the melting point becomes too low. ${ }^{8}$

Figure 2 presents IR spectra of the bulk (unoriented) samples as a function of $r$, which are obtained by using the attenuated total-reflection (ATR) technique. ${ }^{9}$ The spectral region is mainly of the $\mathrm{C}-\mathrm{F}$ stretching vibration $(\mathrm{vC}-\mathrm{F})$ modes. As for the sample of $r=\infty$, polytetrafluoroethylene (PTFE) is employed. Since the band positions are discussed later by comparing to the IP spectrum (Figure 3) that is driven by the transverse-optic (TO; $\operatorname{Im}(\varepsilon)$ ) energy-loss function, ${ }^{9}$ the ATR spectra are all converted to the TO function via the Kramers-Kronig transformation ${ }^{3,9-11}$ using a refractive index of $n=1.35$. $^{12}$ Here, $\varepsilon$ is the complex electric permittivity of the sample.

When we consider that most bands are of the $v C-F$ modes, Figure 2 has unusually too many bands especially for a low $r$. On closer inspection, however, the spectral shape apparently depends on the parameter, $r$ : when $r$ is large, the spectral shape becomes close to the simple spectrum of PTFE. Since a large $r$ means that the normal alkyl part is ignorable, the complex pattern should be induced by the normal alkyl part.

Since the influence of the normal alkyl group on the coupled oscillation of the Rf carbons should propagate in the molecular axis direction, the influence is expected to be separated from the perpendicular components to the axis such as the anti-symmetric and symmetric $\mathrm{CF}_{2}$ stretching vibration $\left(v_{\mathrm{a}} \mathrm{CF}_{2}\right.$ and $v_{\mathrm{s}} \mathrm{CF}_{2}$, respectively) modes, if the molecules are highly oriented on a substrate.

Fortunately, in our previous study, ${ }^{3}$ the compound of $n=$ 9 was found to be aggregated to generate a two-dimensional molecular assembly, in which the molecules are standing perpendicularly at the air-water interface thanks to the carboxylic group. In the present study, the $n=9(r=3.0)$ compound is thus employed to prepare a monolayer on water, and it was transferred onto a silicon substrate, which is a single-monolayer Langmuir-Blodgett (LB) film. ${ }^{13,14}$

To separate the surface-parallel and -perpendicular molecular vibrations, the IR p-polarized multiple-angle incidence resolution spectrometry (pMAIRS) technique ${ }^{15-18}$ is employed. IR pMAIRS generates a set of two absorption spectra of a thin film on a solid substrate. The two spectra are the in-plane (IP) and out-of-plane (OP) spectra, which correspond to the surface-parallel and surface-normal components of a transition moment, respectively. In other words, both spectral pattern of the transmission and reflection-absorption (RA) spectra ${ }^{9,19}$ are simultaneously obtained from an identical sample. When the molecules are perpendicularly oriented on the substrate, the IP and OP spectra correspond to the perpendicular and parallel directions to the "molecular axis," respectively.

The IR pMAIRS spectra of the LB film are presented in Figure 3. The IP spectrum (Figure 3a) is largely simplified from the corresponding bulk spectrum of $r=3.0$ in Figure 2d. For example, the doubly split bands at the apparent positions of 1208 and $1222 \mathrm{~cm}^{-1}$ in Figure 2d are simplified to be a

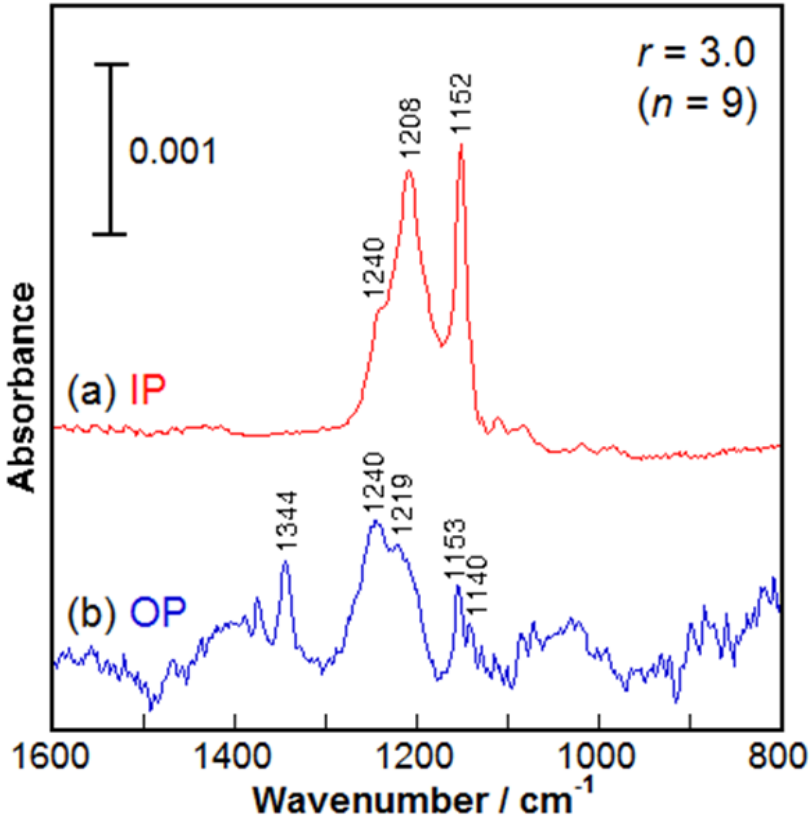

Figure 3 IR pMAIRS spectra of an LB monolayer of the compound of $n=9$ deposited on a silicon substrate.

single band at $1208 \mathrm{~cm}^{-1}$ in Figure 3a. The removed band is attributed to the band at $1219 \mathrm{~cm}^{-1}$ in the OP spectrum (Figure 3b). This highly shifted band should be assigned to the longitudinal-optic (LO) mode of the $v_{\mathrm{a}} \mathrm{CF}_{2}$ band. ${ }^{4}$

The asymmetric broadening of the doublet band in Figure $2 \mathrm{~d}$ is also attributed to the broad band in the OP spectrum at about $1240 \mathrm{~cm}^{-1}$, whose vibrational character is highly complicated. ${ }^{4,7}$ In a similar manner, the many minute bands involving the symmetric $\mathrm{CF}_{3}$ stretching vibration $\left(v_{\mathrm{s}} \mathrm{CF}_{3}\right)$ band $^{3}$ at $1344 \mathrm{~cm}^{-1}$ are removed from the spectrum in Figure $2 \mathrm{~d}$, and instead they appear in the OP spectrum in Figure $3 b$.

The complicated spectrum in Figure $2 \mathrm{~d}$ thus proved to be induced by the molecular vibrations in the direction of the molecular axis (OP). In fact, the band positions (1208 and $1152 \mathrm{~cm}^{-1}$ ) are retained as appeared in the spectrum in Figure $2 d$ thanks to the TO energy-loss function conversion. This supports that the normal alkyl part is the reason why the complicated spectral pattern is generated.

After the removal of the OP contribution, the IP spectrum is remained as fairly similar to the PTFE spectrum in Figure 2e in shape, in which no minute bands appear. PTFE consists of many $\mathrm{CF}_{2}$ groups, in which the terminal $\mathrm{CF}_{3}$ groups can be ignored. No $\mathrm{CF}_{3}$ peak is thus found in the spectrum in Figure 2e. In this manner, as a conclusion, the bands at 1208 and $1152 \mathrm{~cm}^{-1}$ in the IP spectrum are roughly assigned to the $v_{\mathrm{a}} \mathrm{CF}_{2}$ and $v_{\mathrm{s}} \mathrm{CF}_{2}$ bands, respectively. ${ }^{1,3,7}$

Here, we have to pay attention, however, that these bands do not correspond to pure group vibrations, ${ }^{7}$ as opposed to the $v_{\mathrm{a}} \mathrm{CH}_{2}$ and $v_{\mathrm{s}} \mathrm{CH}_{2}$ bands of a normal alkyl group. The transition moments of the $v_{\mathrm{a}} \mathrm{CF}_{2}$ and $\mathrm{v}_{\mathrm{s}} \mathrm{CF}_{2}$ bands are not involved in the surface-parallel plane perfectly, although the molecules are oriented highly perpendicularly, ${ }^{2,3}$ which results in minor bands in the OP spectrum (Figure 3b). This is 
another reason why the $v_{\mathrm{a}} \mathrm{CF}_{2}$ and $\mathrm{v}_{\mathrm{s}} \mathrm{CF}_{2}$ bands are not suitable for analysis of molecular orientation of an $\mathrm{Rf}$ group.

On the other hand, the band at $1344 \mathrm{~cm}^{-1}$ assigned to the $v_{\mathrm{s}} \mathrm{CF}_{3}$ mode is highly useful for the molecular orientation analysis. In the case of a normal alkyl group, the transition moment of the terminal $\mathrm{CH}_{3}$ mode is a little tilted from the molecular axis. In an $\mathrm{Rf}$ group, however, the transition moment of the $v_{\mathrm{s}} \mathrm{CF}_{3}$ mode is directed highly parallel to the molecular axis: the mode is not localized at the $\mathrm{CF}_{3}$ group only, but it is spread over the Rf group especially for a long $\mathrm{Rf}_{\text {group }}{ }^{7}$ due to the coupled oscillation along the Rf group. In this manner, the appearance of the $v_{\mathrm{s}} \mathrm{CF}_{3}$ band in the "OP spectrum only" strongly implies that the $\mathrm{Rf}$ group stands perpendicularly to the monolayer surface, which is consistent with discussion above and other reports. ${ }^{2,3}$

\section{Acknowledgement}

This work was financially supported by a Grant-in-Aid for challenging Exploratory Research (No. 26620118 (TH)) from Japan Society for the Promotion of Science, from Japan Society for the Promotion of Science, the Collaborative Research Program of Institute for Chemical Research, Kyoto University (grant no. 2013-35 (M.S.)), and the "Element Innovation" Project by the Ministry of Education, Culture, Science, Sports and Technology of Japan (MEXT).

\section{References and Notes}

1 M. P. Krafft, J. G. Riess, Chem. Rev. 2009, 109, 1714.

2 K. Honda, M. Morita, H. Otsuka, A. Takahara, Macromolecules 2005, 38, 5699 .

3 T. Hasegawa, T. Shimoaka, N. Shioya, K. Morita, M. Sonoyama, T. Takagi, T. Kanamori, ChemPlusChem 2014, 79, 1421.

4 M. Matsunaga, T. Suzuki, K. Yamamoto and T. Hasegawa, Macromolecules 2008, 41, 5780.

$5 \quad$ E. S. Clark, Polymer 1999,40,4659.

6 L. Piseri, B. M. Powell, G. Dolling, J. Chem. Phys. 1973, 58, 158.

7 C. S. Kim, R. C. Mowrey, J. E. Butler, J. N. Russell, Jr. J. Phys. Chem. B 1998, 102, 9290.

8 H. W. Starkweather, Jr., Macromolecules 1986, 19, 1131.

9 V. P. Tolstoy, I. V. Chernyshova and V. A. Skryshevsky, "Handbook of Infrared Spectroscopy of Ultrathin Films" (John Wiley, Chichester, 2003).

10 P. Yeh, Optical Waves in Layered Media, Wiley-Interscience, Hoboken, 1988.

11 J. S. Plaskett, P. N. Schatz, J. Chem. Phys. 1963, 38, 612.

12 H. K. Kim, F. G. Shi, J. Mat. Sci. 2001, 12, 361.

13 W. D. Harkins, E. Boyd, J. Phys. Chem. 1941, 45, 20.

14 K. B. Blodgett, J. Am. Chem. Soc. 1934, 56, 495.

15 T. Hasegawa, Anal. Chem. 2007, 79, 4385-4389.

16 T. Hasegawa, J. Phys. Chem. B 2002, 106, 4112-4115.

17 T. Hasegawa, Appl. Spectrosc. Rev. 2008, 43, 181-201.

18 N. Shioya, T. Shimoaka, T. Hasegawa Chem. Lett. 2014, 43, 1198.

19 J. Umemura, "Reflection-Absorption Spectroscopy of Thin Films on Metallic Substrates" in Handbook of Vibrational Spectroscopy vol. 2 Eds. J. M. Chalmers and P. R. Griffiths (John Wiley, Chichester, 2002) pp. 982-998. 